\title{
Proposal for Compact Optical Filters Using Large Index Step Binary Supergratings
}

\author{
Farzaneh Afshinmanesh, Student Member, IEEE, Alireza Marandi, Student Member, IEEE, \\ Poman P. M. So, Senior Member, IEEE, and Reuven Gordon, Senior Member, IEEE
}

\begin{abstract}
Compact optical filters are proposed using an aperiodic grating of fixed element size [i.e., a binary supergrating (BSG)] with a large refractive index step. These filters allow for almost arbitrary wavelength filtering, yet they are more compact than previous demonstrations of BSG. The filters are designed using a combination of Boolean particle swarm optimization (B-PSO) and a one-dimensional transfer matrix method. To demonstrate the compact device size, several 50- $\mu \mathrm{m}$-long single-wavelength transmission filters are demonstrated theoretically, each having a different wavelength while using the same structural parameters. A multiwavelength filter is also proposed in an 80- $\mu \mathrm{m}$-long structure to show the versatility of the large refractive index step BSG. A genetic algorithm is substituted for the B-PSO; however, B-PSO shows better performance here. This work may be applied to produce compact optical filters for intrachip optical networks and compact tunable lasers, while using existing single-step photolithography processes.
\end{abstract}

Index Terms-Boolean particle swarm optimization (B-PSO), gratings, intrachip optical networks (ICONs), optical filters, tunable lasers.

\section{INTRODUCTION}

$\mathbf{T}$ HE binary supergrating (BSG) allows for almost arbitrary wavelength filtering [1]. BSG uses an aperiodic structure with a fixed element length and two allowed refractive index values for each element. These constraints make the BSG wellsuited to semiconductor fabrication-requiring only a single lithographic step. So far, the proposed BSG devices have been long $(\sim 1 \mathrm{~mm})$ because they use a small refractive index step, which is the limitation of past design techniques that were based on Fourier methods [1], [2].

Using a large refractive index step allows for more compact optical filters, which is exemplified by photonic bandgap (PBG) structures (e.g., [3]). Single-wavelength transmission filters can be realized by introducing a defect in a PBG structure. Parameters such as the hole diameter and the defect size must be varied with nanometer resolution to accurately vary the transmission wavelength in PBG structures [4]. Alternately, the optical properties of PBG structures can also be tuned by microfluidics [5].

Manuscript received November 20, 2007; revised January 25, 2008. This work was supported by the Natural Sciences and Engineering Research Council of Canada (NSERC).

The authors are with the Department of Electrical and Computer Engineering, University of Victoria, Victoria, BC, V8P 5C2, Canada (e-mail: farzaneh@ece. uvic.ca).

Color versions of one or more of the figures in this letter are available online at http://ieeexplore.ieee.org.

Digital Object Identifier 10.1109/LPT.2008.919600

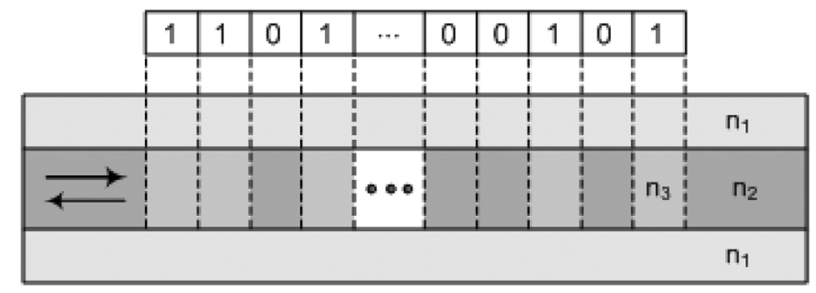

Fig. 1. BSG embedded in an optical waveguide $\left(n_{1}<n_{3}<n_{2}\right)$. A binary string is used to represent the refractive index distribution of the BSG.

In this work, we propose the use of a large refractive index step BSG to allow for compact optical filters that may be readily fabricated with existing photolithography methods. Several compact single-wavelength BSG filters with different transmission wavelengths are presented. The only structural difference between these different filters is the aperiodic order of the refractive index elements, while all the structural parameters, such as element length and refractive index step, are fixed. As compared with defect-based PBG filters, a less stringent fabrication resolution is allowed by the compact large index step BSG, while the device size is still of the order of tens of micrometers. To show the versatility of the large index step BSG, a compact multiwavelength filter is also demonstrated. The compact size of the large refractive index step BSG makes it well-suited for intrachip optical networks (ICONs) [6], [7] and compact tunable lasers.

\section{LARGE REFRACTIVE INDEX STEP BSG}

Fig. 1 shows a schematic of the large refractive index step BSG. The light is confined in the transverse plane by an integrated waveguide. A grating is integrated within the waveguide. The light is impingent normally upon the grating, as shown in Fig. 1 . The grating can be represented by a binary string, where a " 1 " bit corresponds to the small refractive index and a "0" bit corresponds to the large refractive index. Different aperiodic sequences, or binary strings, will have different optical filtering characteristics. The problem of finding the desired sequence for a particular optical filter function cannot be achieved using past Fourier methods. Here, we use stochastic methods to find the desired sequence of the large index step BSG for a desired optical filter.

\section{DESIGN METHOD}

Particle swarm optimization (PSO) applies variations to a population (swarm) of candidate solutions with respect to a given fitness function to approach a desired solution [8]. In this iterative procedure, the specific local solutions are modified 
at each iteration based on the best global solution, the previous-best local solution, and its last modification (i.e., inertia). Due to the binary nature of the BSG problem, we use a binary version of PSO (B-PSO), where Boolean operators replace the conventional PSO operators [9], [10]. B-PSO allows us to design the appropriate device without any a priori knowledge of possible configurations.

The optimization objective is to provide a device with a desired transmittance spectrum, $T_{D}(\lambda)$, in the wavelength range of $\left[\lambda_{1}, \lambda_{2}\right]$. For this purpose, the optimization starts with a set of random binary strings each of them shows a possible BSG configuration. The transmittance spectrum of each candidate BSG, $T_{P}(\lambda)$, is calculated by the transfer matrix method, which relates the reflected and transmitted modes at each interface [11]. The corresponding string to each candidate BSG receives a real number fitness calculated according to transmittance spectra. Many possible fitness functions can be defined to compute the optimization goal. Here, the summation over absolute differences between the desired transmittance spectra and the candidate transmittance spectra is chosen to provide the fitness of each candidate by

$$
F(P)=\sum_{\lambda}\left|T_{P}(\lambda)-T_{D}(\lambda)\right|
$$

After calculating the fitness of all candidates at the first step, the binary strings are updated according to B-PSO rules and this process continues until reaching the optimization goal. The parameter values used are 0.1 for the inertial parameter and 0.5 for the global and local parameters. In each case, we chose the number of solutions at each iteration equal to the total number of elements in the BSG array. Details on B-PSO implementation can be found in [10]. For comparison, we also use a genetic algorithm method in place of the B-PSO. Other design methods are also possible; however, since we seek to demonstrate the large refractive index step BSG capability, a detailed analysis of design methods is not given here.

\section{RESULTS}

\section{A. Wavelength Selection}

First, a series of single-wavelength BSG filters are designed. Each filter has a transmission wavelength that can be selectively varied over a $10-\mathrm{nm}$ wavelength range, while all the structural parameters such as element length, number of elements, and refractive index step are maintained. The desired wavelength is selected by finding the correct order for the binary sequence.

Fig. 2 shows the transmittance spectra of the optimized BSG filters in the wavelength range of $1540-1550 \mathrm{~nm}$ with 2-nm peak spacing. The target wavelengths are shown with dashed vertical lines. The optimization goal was to provide maximum transmission at the desired wavelength while minimizing transmission at other wavelengths with a fitness function defined in (1). The refractive indexes are 3.5 and 2.7. The element length is $50 \mathrm{~nm}$, which is the same as past work [2]. The number of elements is 1000 and the total length of each filter is $50 \mu \mathrm{m}$,

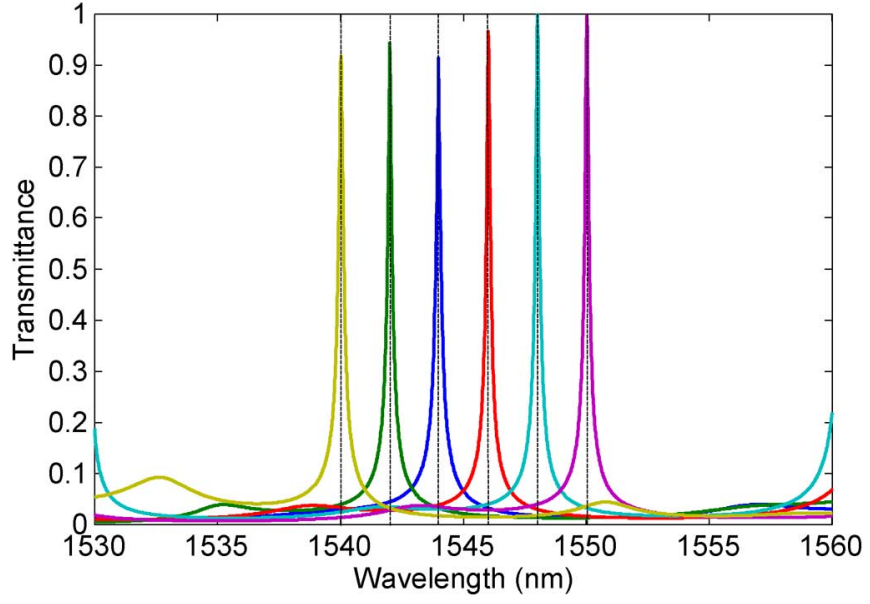

Fig. 2. Transmittance spectra of 50- $\mu \mathrm{m}$-long BSG filters. The peak spacing is $2 \mathrm{~nm}$.

\section{A.}

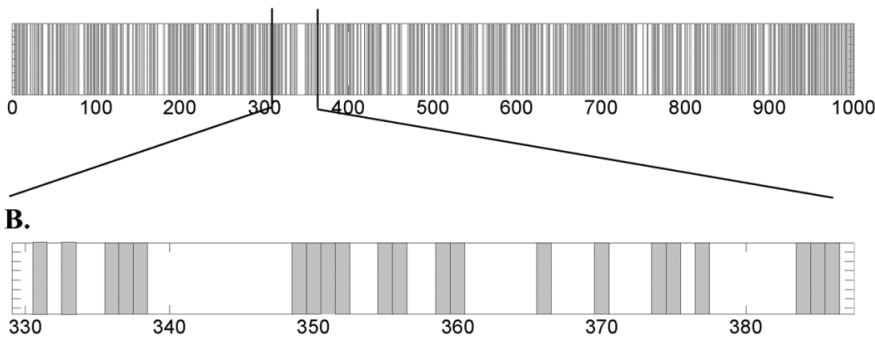

Fig. 3. (A) Refractive index distribution of the BSG filter at $1550 \mathrm{~nm}$ with 1000 elements. Small refractive index is shown with a dark color bar and large refractive index with a light color bar. (B) A magnified region of refractive index distribution in part A.

accordingly. It is clear that the B-PSO is able to achieve good single-wavelength selection over a wide range of wavelengths.

Fig. 3 shows the refractive index distribution of the optimized BSG filter at $1550 \mathrm{~nm}$, with the transmission shown in Fig. 2. A blown-up portion of this distribution is shown to emphasize the aperiodic nature of the optimized BSG structure.

\section{B. Multiwavelength Filter}

In this section, a multiwavelength filter with three equally spaced peaks of equal transmission is presented. The wavelength spacing was chosen to be $4 \mathrm{~nm}$, as in past works on filters for tunable lasers [12]. Here, the element length is doubled to $100 \mathrm{~nm}$ (as compared with the single-wavelength filter) to enable less demanding fabrication resolution. It is desirable to maintain a compact design, at least below $100 \mu \mathrm{m}$. Therefore, the number of elements was reduced to 800 , so that the overall length was $80 \mu \mathrm{m}$. The corresponding size of the search space is $2^{800}$. The refractive indexes are chosen to be 3.5 and 2.5 .

In this case, the optimization of (1) has three times the number of wavelengths as the previous example. Therefore, computation time is increased considerably. As a result, we added an ad hoc penalty to the fitness defined in (1) to wean initial poor candidates from the search space. 


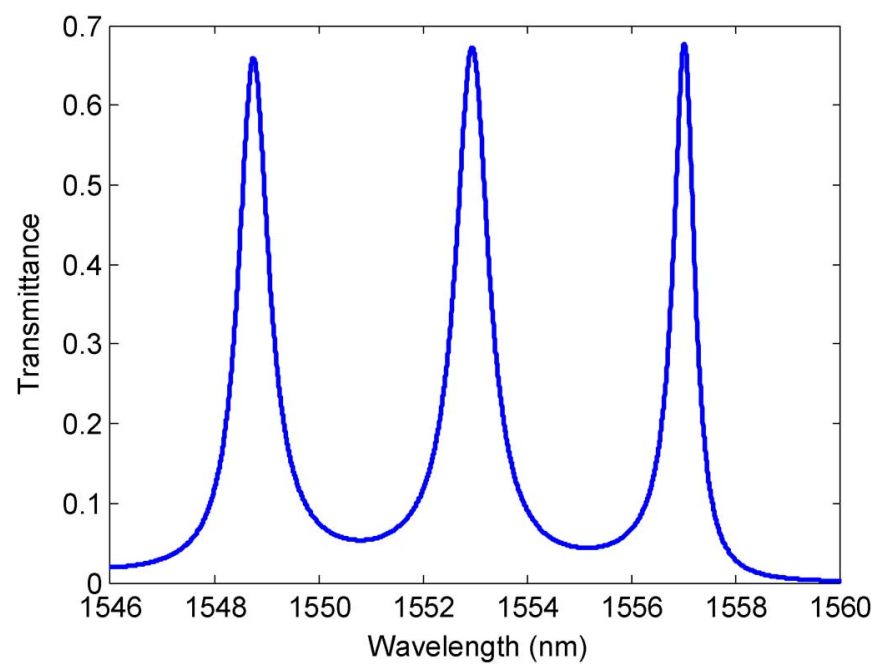

Fig. 4. Transmittance spectrum of an 80- $\mu \mathrm{m}$-long multiwavelength BSG filter.

Fig. 4 shows the results for the calculated transmittance spectrum of the optimized BSG.

\section{DISCUSSION}

The details of BSG implementation depend upon the specific material system used-a tunable laser will likely use active semiconductor materials, whereas an ICON will likely use silicon-based materials. Here, we do not wish to limit the consideration to a specific implementation, so the problems are considered in one dimension, as was done in the past [1], [2], with the understanding that diffraction losses should be minimized.

As demonstrated in Fig. 4, the large refractive index step BSG provides multiwavelength filters that are an order of magnitude more compact than past multiwavelength gratings in integrated tunable laser technologies [2], [13]. As compared with single-wavelength PBG filters, the large refractive index step BSG has relaxed resolution, which allows for single-step photolithography, while retaining a compact structure. It should be noted that the desired filter may not exist within the search space provided by the large refractive index step BSG, and so we only claim near-arbitrary filtering capability. While the phase was not included in the fitness function defined in (1), it may be modified in a straightforward way to include phase filtering as well.

We have compared the performance of the B-PSO with GA in above problems. Using a multipoint crossover GA with a mutation rate of 0.2 and number of elite solutions equal to 20 , it is found that B-PSO converged to a better result in a shorter time. Although in many cases both algorithms result in good solutions, the simpler implementation and reduced bookkeeping of PSO make it appealing [14]. Past work has demonstrated convergence guaranteed PSO [15], [16], which may be used to further improve the performance of the compact optical filters proposed here.

\section{CONCLUSION}

Compact optical filters are demonstrated by using a large refractive index step BSG structure. Wavelength selection in single wavelength filters has been demonstrated without changing the refractive index step or element length. A multiwavelength filter which is an order of magnitude shorter than previous BSG devices has also been presented. The BSG architecture may be readily fabricated with optical lithography and existing semiconductor technology. The large refractive index step BSG allows for compact devices that can be densely integrated, which is promising for many applications, including ICONs and compact tunable lasers.

\section{REFERENCES}

[1] D. Levner, M. F. Fay, and J. M. Xu, "Programmable spectral design using a simple binary Bragg-diffractive structure," IEEE J. Quantum Electron., vol. 42, no. 4, pp. 410-417, Apr. 2006.

[2] I. A. Avrutsky, D. S. Ellis, A. Tager, H. Anis, and J. M. Xu, "Design of widely tunable semiconductor lasers and the concept of binary superimposed gratings (BSG's)," IEEE J. Quantum Electron., vol. 34, no. 4, pp. 729-741, Apr. 1998.

[3] J. S. Foresi, P. R. Villeneuve, J. Ferrera, E. R. Thoen, G. Steinmeyer, S. Fan, J. D. Joannopoulos, L. C. Kimerling, H. I. Smith, and E. P. Ippen, "Photonic-bandgap microcavities in optical waveguides," Nature, vol. 390, pp. 143-145, Nov. 1997.

[4] D. J. Ripin, K. Lim, G. S. Petrich, P. R. Villeneuve, S. Fan, E. R. Thoen, J. D. Joannopoulos, E. P. Ippen, and L. A. Kolodziejski, "Onedimensional photonic bandgap microcavities for strong optical confinement in GaAs and $\mathrm{GaAs} / \mathrm{Al}_{x} \mathrm{O}_{y}$ semiconductor waveguides," $J$. Lightw. Technol., vol. 17, no. 11, pp. 2152-2160, Nov. 1999.

[5] A. Sharkawy, D. Pustai, S. Shi, D. W. Prather, S. McBride, and P. Zanzucchi, "Modulating dispersion properties of low index photonic crystal structures using microfluidics," Opt. Express, vol. 11, no. 20, pp. 2589-2596, Sep. 2003

[6] J. A. Kash, "Intrachip optical networks for a future supercomputer-on-a chip," Photon. Switching, pp. 55-56, 2007.

[7] F. Xia, M. Rooks, L. Sekaric, and Y. Vlosov, "Ultra-compact high order ring resonator filters using submicron silicon photonic wires for on-chip optical interconnects," Opt. Express, vol. 15, no. 19, pp. 11934-11941, Sep. 2005.

[8] S. Mei-Ping and G. Guo-Chang, "Research on particle swarm optimization: A review," in Proc. Int. Conf. Machine Learning and Cybernetics, 2004, vol. 4, pp. 2236-2241.

[9] F. Afshinmanesh, A. Marandi, and M. Shahabadi, "Design of a single-feed dual-band dual-polarized printed microstrip antenna using a Boolean particle swarm optimization," IEEE Trans. Antennas Propag., accepted for publication.

[10] A. Marandi, F. Afshinmanesh, M. Shahabadi, and F. Bahrami, "Boolean particle swarm optimization and its application to the design of a dual-band dual-polarized printed antenna," in Proc. IEEE Congr. Evolutionary Computation, 2006, pp. 3212-3218.

[11] L. A. Coldren and S. W. Corzine, Diode Lasers and Photonic Integrated Circuits. New York: Wiley-Interscience, 1995.

[12] B. Mason, S. P. DenBaars, and L. A. Coldren, "Tunable sampled-grating DBR lasers with integrated wavelength monitors," IEEE Photon. Technol. Lett., vol. 10, no. 8, pp. 1085-1087, Aug. 1998.

[13] A. J. Ward, D. J. Robbins, G. Busico, E. Barton, L. Ponnampalam, J. P. Duck, N. D. Whitbread, P. J. Williams, D. C. J. Reid, A. C. Carter, and M. J. Wale, "Widely tunable DS-DBR laser with monolithically integrated SOA: Design and performance," IEEE J. Quantum Electron., vol. 11, no. 1, pp. 149-156, Jan./Feb. 2005.

[14] D. W. Boeringer and D. H. Werner, "Particle swarm optimization versus genetic algorithms for phased array synthesis," IEEE Trans. Antennas Propag., vol. 52, no. 3, pp. 771-779, Mar. 2004.

[15] J. Sun, W. Xu, and B. Feng, "Adaptive parameter control for quantum-behaved particle swarm optimization on individual level," in Proc. IEEE Int. Conf. Systems, Man, and Cybernetics, 2005, pp. 3049-3054.

[16] X. Chen and Y. Li, "A modified PSO structure resulting in high exploration ability with convergence guaranteed," IEEE Trans. Syst., Man, Cybern., vol. 37, no. 5, pp. 1271-1289, Oct. 2007. 